PROCEEDINGS OF THE

AMERICAN MATHEMATICAL SOCIETY

Volume 139, Number 11, November 2011, Pages 3973-3983

S 0002-9939(2011)11185-7

Article electronically published on May 25, 2011

\title{
HOMOCLINIC ORBITS OF SUPERLINEAR HAMILTONIAN SYSTEMS
}

\author{
GUANWEI CHEN AND SHIWANG MA
}

(Communicated by Matthew J. Gursky)

ABSTRACT. In this paper, we consider the first-order Hamiltonian system

$$
J \dot{u}(t)+\nabla H(t, u(t))=0, \quad t \in \mathbb{R} .
$$

Here the classical Ambrosetti-Rabinowitz superlinear condition is replaced by a general super-quadratic condition. We will study the homoclinic orbits for the system. The main idea here lies in an application of a variant generalized weak linking theorem for a strongly indefinite problem developed by Schechter and Zou.

\section{INTRODUCTION AND MAIN RESULT}

Let us consider the first-order Hamiltonian system

$$
J \dot{u}(t)+\nabla H(t, u(t))=0, \quad t \in \mathbb{R},
$$

where $H: \mathbb{R} \times \mathbb{R}^{2 n} \rightarrow \mathbb{R}$ is $T$-periodic in its first variable, and

$$
J=\left(\begin{array}{cc}
0 & -I_{n} \\
I_{n} & 0
\end{array}\right)
$$

denotes the standard symplectic matrix and $\nabla H(t, u)$ denotes its gradient with respect to the $u$ variable. Suppose that

$$
H(t, u)=\frac{1}{2} L(t)(u, u)+W(t, u),
$$

where $(\cdot, \cdot)$ denotes the inner product in $\mathbb{R}^{2 n}$ and $L \in C^{1}\left(\mathbb{R}, \mathbb{R}^{4 n^{2}}\right)$ is a $2 n \times 2 n$ symmetric matrix-valued function and $W \in C^{1}\left(\mathbb{R} \times \mathbb{R}^{2 n}, \mathbb{R}\right)$. Recall that a solution $u$ of (1.1) is a homoclinic orbit if $u \neq 0$ and $u \rightarrow 0$ as $|t| \rightarrow \infty$. In this paper we study the existence of homoclinic orbits of (1.1), which are ground state solutions of the system.

The existence and multiplicity of homoclinic orbits of (1.1) have been studied extensively via modern variational methods under different assumptions. We outline them as follows. Many authors (see [1, 2, 4, 15, 8, 11, 12, 17, 20, 24, and so on) have studied the existence of homoclinic orbits for the system (1.1) in the cases

Received by the editors September 17, 2010.

2010 Mathematics Subject Classification. Primary 37J45, 37K05, 58E05.

Key words and phrases. Homoclinic orbit, first-order Hamiltonian system, ground state solution, concentration compactness principle.

Research supported by the Specialized Fund for the Doctoral Program of Higher Education and the National Natural Science Foundation of China.

(C)2011 American Mathematical Society Reverts to public domain 28 years from publication 
when $H(t, u)$ depends periodically on $t$ by considering the following super-quadratic condition: there exists a constant $\mu>2$ such that

$$
0<\mu W(t, x) \leq(\nabla W(t, x), x), \quad x \in \mathbb{R}^{2 n} \backslash\{0\},
$$

which is now known as the Ambrosetti-Rabinowitz super-quadratic condition (A-R condition for short).

If $H(t, u)$ depends periodically on $t$ such that 0 lies in a gap of $\sigma(B)$, the spectrum of $B:=-J\left(\frac{d}{d t}\right)-L(t)$, and if in addition $W(t, u)$ is super-quadratic or asymptotically quadratic in $u$ at infinity, then [6] obtains infinitely many homoclinic orbits. If $H(t, u)$ depends periodically on $t$ while 0 lies on the boundary of $\sigma(B)$, considering some super-quadratic system, then [5] obtains one homoclinic orbit and [4] obtains infinitely many homoclinic orbits.

The first to study the existence of homoclinic orbits of (1.1) by means of critical point theory were Coti-Zelati, Ekeland and Séré [2]. They assumed that $L$ is constant with 0 a hyperbolic point of the Hamiltonian operator $B, W(t, u)$ strictly convex in $u$ and satisfying the Ambrosetti-Rabinowitz (super-quadratic) growth condition. They proved the existence and multiplicity of homoclinic orbits of (1.1). This result was deepened in [11, 12] when Séré established the existence of infinitely many homoclinic orbits. Subsequently, Hofer and Wysocki [8], using Fredholm operator theory and a linking argument, and Tanaka [17, passing through a subharmonic approach, managed to remove the convexity assumption to get one homoclinic orbit. Later linking-type arguments were used in [1, 4, 5, to show the existence and multiplicity of homoclinic orbits of (1.1) when $L$ depends periodically on $t$ and certain symmetries on $W(t, u)$ are assumed for the multiplicity.

In our paper, the classical A-R condition on $\nabla W$ is replaced by a general superquadratic condition, and we are interested in the existence of a homoclinic orbit, which is a ground state solution of (1.1), i.e., a solution corresponding to the least energy of the action functional of (1.1). We should mention that some results concerning the existence of ground state solutions for Schrödinger equations have been obtained by [16, 23. Our main idea lies in the application of a variant generalized weak linking theorem for a strongly indefinite problem developed by Schechter and Zou [15. See also [14, 19, where the authors developed the idea of a Monotonicity Trick for strongly indefinite problems; the original idea is due to 9, 13. Note that some authors have studied several different problems by the same method as our paper; see [3, 21, 22, 23] and the references therein. Among these problems are the second-order Hamiltonian system without A-R condition and spectrum zero [3], the discrete Schrödinger equation with spectrum zero [21, the Schrödinger equation with spectrum zero [22] and the Schrödinger equation without spectrum zero 23.

Let $B:=-J\left(\frac{d}{d t}\right)-L(t)$. In order to state the main results, we assume that $L(t)$ and $W(t, u)\left(t \in \mathbb{R}, u \in \mathbb{R}^{2 n}\right)$ satisfy the following assumptions:

$\left(L_{0}\right) L(t)$ is $T$-periodic in $t$ such that 0 lies in a gap of $\sigma(B)$, that is, $\underline{\Lambda}:=\sup (\sigma(B) \cap(-\infty, 0))<0<\bar{\Lambda}:=\inf (\sigma(B) \cap(0, \infty))$.

$\left(W_{0}\right) \quad(\nabla W(t, u), v)(u, v) \geq 0$ uniformly in $t$.

$\left(W_{1}\right) \quad|\nabla W(t, u)| \leq a\left(1+|u|^{p-1}\right)$ for some $a>0$ and $p \in(2, \infty)$.

$\left(W_{2}\right) \quad|\nabla W(t, u)|=o(|u|)$ as $|u| \rightarrow 0$ uniformly in $t$.

$\left(W_{3}\right) \frac{W(t, u)}{|u|^{2}} \rightarrow \infty$ as $|u| \rightarrow \infty$ uniformly in $t$. 
$\left(W_{4}\right) \frac{1}{2}(\nabla W(t, u), u)>W(t, u)$ for all $u \in \mathbb{R}^{2 n} \backslash\{0\}, W(t, u) \geq 0$ for all $u \in \mathbb{R}^{2 n}$.

$\left(W_{5}\right) W(t, u)=W(t, v)$ and $(\nabla W(t, u), v) \leq(\nabla W(t, u), u)$ uniformly in $t$ if $|u|=|v|$.

$\left(W_{6}\right) \quad(\nabla W(x, u), v) \neq(\nabla W(t, v), u)$ for any $t \in \mathbb{R}$ if $|u| \neq|v|$ and $(u, v) \neq 0$.

As is shown in the next examples, our assumptions are reasonable and there are cases in which the well-known Ambrosetti-Rabinowitz super-quadratic condition is not satisfied.

Example 1.1. Let $W(t, u)=|u|^{p}$, where $p>2$. Clearly, $W(t, u)$ satisfies $\left(W_{0}\right)-$ $\left(W_{6}\right)$ and the well-known Ambrosetti-Rabinowitz super-quadratic condition.

Example 1.2. Let $W(t, u)=g(t)\left(|u|^{p}+(p-2)|u|^{p-\varepsilon} \sin ^{2}\left(\frac{|u|^{\varepsilon}}{\varepsilon}\right)\right)$, where $g(t)>$ 0 is $T$-periodic in $t, 0<\varepsilon<p-2$ and $p$ is the parameter in $\left(W_{1}\right)$. Note that

$$
\begin{aligned}
& \nabla W(t, u) \\
& =g(t) u\left[(p-2)(p-\varepsilon)|u|^{p-\varepsilon-2} \sin ^{2}\left(\frac{|u|^{\varepsilon}}{\varepsilon}\right)+\left(p+(p-2) \sin \left(\frac{2|u|^{\varepsilon}}{\varepsilon}\right)\right)|u|^{p-2}\right] .
\end{aligned}
$$

It is not hard to check that $W(t, u)$ satisfies $\left(W_{0}\right)-\left(W_{6}\right)$. However, similar to Remark 1.2 of [19], let $u_{m}=\left(\varepsilon\left(m \pi+\frac{3 \pi}{4}\right)\right)^{\frac{1}{\varepsilon}} L_{2 n}$, where $L_{2 n}=(1,0, \ldots, 0)$. Then for any $\gamma>2$, one has

$$
\begin{aligned}
\left(\nabla W\left(t, u_{m}\right), u_{m}\right)-\gamma W\left(t, u_{m}\right) \\
=g(t)\left[(p-\gamma)\left|u_{m}\right|^{p}+(p-2)(p-\varepsilon-\gamma)\left|u_{m}\right|^{p-\varepsilon} \sin ^{2}\left(\frac{\left|u_{m}\right|^{\varepsilon}}{\varepsilon}\right)\right] \\
+g(t)(p-2)\left|u_{m}\right|^{p} \sin 2\left(\left|u_{m}\right|^{\varepsilon} / \varepsilon\right) \\
=g(t)\left|u_{m}\right|^{p}\left[2-\gamma+\frac{(p-2)(p-\varepsilon-\gamma) \sin ^{2}\left(\frac{\left|u_{m}\right|^{\varepsilon}}{\varepsilon}\right)}{\left|u_{m}\right|^{\varepsilon}}\right] \\
\rightarrow-\infty \text { as } m \rightarrow \infty .
\end{aligned}
$$

That is, the condition (1.2) cannot be satisfied for $\gamma>2$.

Our main result reads as follows:

Theorem 1.3. If assumptions $\left(L_{0}\right)$ and $\left(W_{0}\right)-\left(W_{6}\right)$ are satisfied, then system (1.1) has at least one homoclinic orbit, and it is a ground state solution of (1.1).

The rest of this paper is organized as follows. In Section 2, we give some preliminary results which are needed in later sections. In Section 3, we give the detailed proof of our main result.

\section{Preliminary lemmas}

On $E:=H^{1}\left(\mathbb{R}, \mathbb{R}^{2 n}\right)$ we define the functional

$$
I(u)=\frac{1}{2}(B u, u)-\psi(u)
$$


where $\psi(u)=\int_{\mathbb{R}} W(t, u(t)) d t$. The hypotheses on $W$ imply that $I$ and $\psi$ are continuously differentiable, and for $\forall u, v \in E$ we have

$$
\begin{gathered}
\left\langle I^{\prime}(u), v\right\rangle=(B u, v)-\int_{\mathbb{R}}(\nabla W(t, u), v) d t, \\
\left\langle\psi^{\prime}(u), v\right\rangle=\int_{\mathbb{R}}(\nabla W(t, u), v) d t .
\end{gathered}
$$

Throughout this paper we denote by $\|\cdot\|_{q}$ the usual $L^{q}$-norm, and we set $B_{r}(s):=$ $[s-r, s+r]$. Under assumptions $\left(L_{0}\right), B=-J \frac{d}{d t}-L(t)$ is a selfadjoint operator acting on $L^{2}\left(\mathbb{R}, \mathbb{R}^{2 n}\right)$ with domain $D(B):=H^{1}\left(\mathbb{R}, \mathbb{R}^{2 n}\right)$, and we have the orthogonal decomposition

$$
L^{2}=L^{2}\left(\mathbb{R}, \mathbb{R}^{2 n}\right)=L^{-} \oplus L^{+}, \quad u=u^{-}+u^{+}
$$

such that $B$ is negative (resp., positive) in $L^{-}$(resp., in $L^{+}$). Let $E:=D\left(|B|^{1 / 2}\right.$ ) be a Hilbert space equipped with the inner product

$$
\langle u, v\rangle=\left(|B|^{1 / 2} u,|B|^{1 / 2} v\right)_{L^{2}}
$$

and norm $\|u\|=\langle u, u\rangle^{\frac{1}{2}}$, where $(\cdot, \cdot)_{L^{2}}$ denotes the inner product of $L^{2}$. We have the decomposition

$$
E=E^{-} \oplus E^{+}, \quad \text { where } E^{ \pm}=E \cap L^{ \pm},
$$

orthogonal with respect to both $(\cdot, \cdot)_{L^{2}}$ and $\langle\cdot, \cdot\rangle$.

On $E$ we define the functional (2.1) in another form:

$$
\begin{aligned}
I(u) & =\frac{1}{2}(B u, u)-\int_{\mathbb{R}} W(t, u) d t \\
& =\frac{1}{2}\left(\left\|u^{+}\right\|^{2}-\left\|u^{-}\right\|^{2}\right)-\int_{\mathbb{R}} W(t, u) d t .
\end{aligned}
$$

The hypotheses on $W$ imply that $I \in C^{1}(E, \mathbb{R})$, and a standard argument shows that critical points of $I$ are homoclinic orbits of (1.1).

The following abstract critical point theorem plays an important role in proving our main result. Let $E$ be a Hilbert space with norm $\|\cdot\|$ and having an orthogonal decomposition $E=N \oplus N^{\perp}$, where $N \subset E$ is a closed and separable subspace. There exists a norm $|v|_{\omega}$ satisfying $|v|_{\omega} \leq\|v\|$ for all $v \in N$ and inducing a topology equivalent to the weak topology of $N$ on bounded subsets of $N$. For $u=v+w \in$ $E=N \oplus N^{\perp}$ with $v \in N, w \in N^{\perp}$, we define $|u|_{\omega}^{2}=|v|_{\omega}^{2}+\|w\|^{2}$. In particular, if $\left(u_{n}=v_{n}+w_{n}\right)$ is $|\cdot|{ }_{\omega}$-bounded and $u_{n} \stackrel{|\cdot|_{\omega}}{\rightarrow} u$, then $v_{n} \rightarrow v$ weakly in $N, w_{n} \rightarrow w$ strongly in $N^{\perp}, u_{n} \rightarrow v+w$ weakly in $E$ (cf. [15).

Let $E=E^{-} \oplus E^{+}, z_{0} \in E^{+}$with $\left\|z_{0}\right\|=1$. Let $N:=E^{-} \oplus \mathbb{R} z_{0}$ and $E_{1}^{+}:=$ $N^{\perp}=\left(E^{-} \oplus \mathbb{R} z_{0}\right)^{\perp}$. For $R>0$, let

$$
Q:=\left\{u:=u^{-}+s z_{0}: s \in \mathbb{R}^{+}, u^{-} \in E^{-},\|u\|<R\right\}
$$

with $p_{0}=s_{0} z_{0} \in Q, s_{0}>0$. We define

$$
D:=\left\{u:=s z_{0}+w^{+}: s \geq 0, w^{+} \in E_{1}^{+},\left\|s z_{0}+w^{+}\right\|=s_{0}\right\} .
$$


For $I \in C^{1}(E, \mathbb{R})$, define $\Gamma:=\left\{h \mid h:[0,1] \times \bar{Q} \mapsto E\right.$ is $|\cdot|_{\omega}$-continuous, $h(0, u)=$ $u, I(h(s, u)) \leq I(u), \forall u \in \bar{Q} ;$ for any $\left(s_{0}, u_{0}\right) \in[0,1] \times \bar{Q}$, there is a $|\cdot| \omega_{\omega^{-}}$ neighborhood $U_{\left(s_{0}, u_{0}\right)}$, such that $\left\{u-h(t, u):(t, u) \in U_{\left(s_{0}, u_{0}\right)} \cap([0,1] \times \bar{Q})\right\} \subset$ $\left.E_{f i n}.\right\}$, where $E_{f i n}$ denotes various finite-dimensional subspaces of $E, \Gamma \neq 0$ since $i d \in \Gamma$.

Lemma $2.1([7])$. E embeds continuously into $H^{\frac{1}{2}}\left(\mathbb{R}, \mathbb{R}^{2 n}\right)$; hence, $E$ embeds continuously into $L^{q}$ and compactly into $L_{l o c}^{q}$ for all $q \in[2, \infty)$.

The variant weak linking theorem is:

Lemma 2.2 ([15]). The family of $C^{1}$-functionals $\left\{I_{\lambda}\right\}$ has the form

$$
I_{\lambda}(u):=J(u)-\lambda K(u), \quad \forall \lambda \in[1,2] .
$$

Assume that:

(1) $K(u) \geq 0, \forall u \in E, I_{1}=I$.

(2) $J(u) \rightarrow \infty$ or $K(u) \rightarrow \infty$ as $\|u\| \rightarrow \infty$.

(3) $I_{\lambda}$ is $|\cdot|_{\omega}$-upper semicontinuous and $I_{\lambda}^{\prime}$ is weakly sequentially continuous on E. Moreover, $I_{\lambda}$ maps bounded sets to bounded sets.

(4) $\sup _{\partial Q} I_{\lambda}<\inf _{D} I_{\lambda}, \forall \lambda \in[1,2]$.

Then for almost all $\lambda \in[1,2]$, there exists a sequence $\left\{u_{n}\right\}$ such that

$$
\sup _{n}\left\|u_{n}\right\|<\infty, \quad I_{\lambda}^{\prime}\left(u_{n}\right) \rightarrow 0, \quad I_{\lambda}\left(u_{n}\right) \rightarrow c_{\lambda},
$$

where $c_{\lambda}:=\inf _{h \in \Gamma} \sup _{u \in Q} I_{\lambda}(h(1, u)) \in\left[\inf _{D} I_{\lambda}, \sup _{\bar{Q}} I\right]$.

In order to apply Lemma 2.2 , we consider

$$
I_{\lambda}(u):=\frac{1}{2}\left\|u^{+}\right\|^{2}-\lambda\left(\frac{1}{2}\left\|u^{-}\right\|^{2}+\int_{\mathbb{R}} W(t, u) d t\right) .
$$

It is easy to see that $I_{\lambda}$ satisfies conditions $(a),(b)$ in Lemma 2.2. To see $(c)$, if $u_{n} \stackrel{|\cdot| \omega}{\rightarrow} u$, then $u_{n}^{+} \rightarrow u^{+}$and $u_{n}^{-} \rightarrow u^{-}$in $E$; going to a subsequence if necessary, $u_{n} \rightarrow u$ a.e. on $\mathbb{R}$. Using Fatou's lemma and the weak lower semicontinuity of the norm, we have

$$
\varlimsup_{n \rightarrow \infty} I_{\lambda}\left(u_{n}\right) \leq I_{\lambda}(u),
$$

which means that $I_{\lambda}$ is $|\cdot|_{\omega}$-upper semicontinuous. $I_{\lambda}^{\prime}$ is weakly sequentially continuous on $E$ due to [18. To continue the discussion, we still need to verify condition $(d)$.

Lemma 2.3. Under assumptions $\left(L_{0}\right)$ and $\left(W_{0}\right)-\left(W_{6}\right)$, the following hold:

(1) There exists $\rho>0$ independent of $\lambda \in[1,2]$ such that $\kappa:=\inf I_{\lambda}\left(S_{\rho} E^{+}\right)>$ 0 , where $S_{\rho} E^{+}:=\left\{z \in E^{+}:\|z\|=\rho\right\}$.

(2) For fixed $z_{0} \in E^{+}$with $\left\|z_{0}\right\|=1$ and any $\lambda \in[1,2]$, there is $R>\rho>0$ such that $\sup I_{\lambda}(\partial Q) \leq 0$, where $Q:=\left\{u:=v+s z_{0}: s \geq 0, v \in E^{-},\|u\|<R\right\}$.

Proof. (1) Under assumptions $\left(W_{1}\right)$ and $\left(W_{2}\right)$, we know that for any $\varepsilon>0$ there exists $C_{\varepsilon}>0$ such that

$$
|\nabla W(t, u)| \leq \varepsilon|u|+C_{\varepsilon}|u|^{p-1},
$$

which together with $\left(W_{4}\right)$ implies that

$$
|W(t, u)| \leq \varepsilon|u|^{2}+C_{\varepsilon}|u|^{p} .
$$


Hence, for any $u \in E^{+}$,

$$
I_{\lambda}(u) \geq \frac{1}{2}\|u\|^{2}-\lambda \varepsilon\|u\|^{2}-C_{\varepsilon}^{\prime}\|u\|^{p},
$$

which implies the conclusion.

(2) The proof for the case $\lambda=1$ is contained in [16]; we outline it here for the completeness of the paper. Suppose by contradiction that there exist $u_{n} \in$ $E^{-} \oplus \mathbb{R}^{+} z_{0}$ such that $I_{\lambda}\left(u_{n}\right)>0$ for all $n$ and $\|u\| \rightarrow \infty$ as $n \rightarrow \infty$. Set $w_{n}=$ $\frac{u_{n}}{\left\|u_{n}\right\|}=s_{n} z_{0}+w_{n}^{-}$. Then

$$
0<\frac{I_{\lambda}\left(u_{n}\right)}{\left\|u_{n}\right\|^{2}}=\frac{1}{2}\left(s_{n}^{2}-\lambda\left\|w_{n}^{-}\right\|^{2}\right)-\lambda \int_{\mathbb{R}} \frac{W\left(t, u_{n}\right)}{u_{n}^{2}} w_{n}^{2} d t .
$$

From $\left(W_{4}\right)$, we know that $W(t, u) \geq 0$ and we have

$$
\left\|w_{n}^{-}\right\|^{2} \leq \lambda\left\|w_{n}^{-}\right\|^{2}<s_{n}^{2}=1-\left\|w_{n}^{-}\right\|^{2} ;
$$

therefore $\left\|w_{n}^{-}\right\| \leq \frac{1}{\sqrt{2}}$ and $1-\frac{1}{\sqrt{2}} \leq s_{n} \leq 1$.

So $s_{n} \rightarrow s \neq 0$ after passing to a subsequence, $w_{n} \rightarrow w$ and $w_{n} \rightarrow w$ a.e. in $\mathbb{R}$. Hence $w=s z_{0}+w^{-} \neq 0$ and, since $\left|u_{n}\right| \rightarrow \infty$ if $w \neq 0$, it follows from $\left(W_{3}\right)$ and Fatou's lemma that

$$
\int_{\mathbb{R}} \frac{W\left(t, u_{n}\right)}{u_{n}^{2}} w_{n}^{2} d t \rightarrow \infty
$$

which contradicts (2.5). The proof is finished.

Applying Lemma 2.2, we soon obtain the following facts:

Lemma 2.4. Under assumptions $\left(L_{0}\right)$ and $\left(W_{0}\right)-\left(W_{6}\right)$, for almost all $\lambda \in[1,2]$, there exists a sequence $\left\{u_{n}\right\}$ such that

$$
\sup _{n}\left\|u_{n}\right\|<\infty, \quad I_{\lambda}^{\prime}\left(u_{n}\right) \rightarrow 0, \quad I_{\lambda}\left(u_{n}\right) \rightarrow c_{\lambda} \in\left[\kappa, \sup _{\bar{Q}} I\right] .
$$

Lemma 2.5. Under assumptions $\left(L_{0}\right)$ and $\left(W_{0}\right)-\left(W_{6}\right)$, for almost all $\lambda \in[1,2]$, there exists $a u_{\lambda}$ such that

$$
I_{\lambda}^{\prime}\left(u_{\lambda}\right)=0, \quad I_{\lambda}\left(u_{\lambda}\right) \leq \sup _{\bar{Q}} I .
$$

Proof. Let $\left\{u_{n}\right\}$ be the sequence obtained in Lemma 2.4, write $u_{n}=u_{n}^{-}+u_{n}^{+}$with $u_{n}^{ \pm} \in E^{ \pm}$. Since $\left\{u_{n}\right\}$ is bounded, we have that either $u_{n}^{+}$is vanishing, i.e.,

$$
\lim _{n \rightarrow \infty} \sup _{s \in \mathbb{R}} \int_{B_{1}(s)}\left(u_{n}^{+}\right)^{2} d t=0,
$$

or is nonvanishing; i.e., there exist $r, \delta>0$ and a sequence $s_{n} \in \mathbb{R}$ such that

$$
\lim _{n \rightarrow \infty} \int_{B_{r}\left(s_{n}\right)}\left(u_{n}^{+}\right)^{2} d t \geq \delta
$$

If $u_{n}^{+}$is vanishing, by Lion's concentration compactness principle [10, we have that $u_{n}^{+} \rightarrow 0$ in $L^{q}\left(\mathbb{R}, \mathbb{R}^{2 n}\right)$ for all $q \in(2, \infty)$. By Hölder's inequality and (2.3), we know that

$$
\int_{\mathbb{R}}\left|\left(\nabla W\left(t, u_{n}\right), u_{n}^{+}\right)\right| d t \leq \varepsilon \int_{\mathbb{R}}\left|u_{n}\right| \cdot\left|u_{n}^{+}\right| d t+C_{\varepsilon} \int_{\mathbb{R}}\left|u_{n}\right|^{p-1}\left|u_{n}^{+}\right| d t \rightarrow 0 .
$$


Therefore,

$$
I_{\lambda}\left(u_{n}\right) \leq\left\|u_{n}^{+}\right\|^{2}=I_{\lambda}^{\prime}\left(u_{n}\right) u_{n}^{+}+\lambda \int_{\mathbb{R}}\left(\nabla W\left(t, u_{n}\right), u_{n}^{+}\right) d t \rightarrow 0
$$

this contradicts the fact that $I_{\lambda}\left(u_{n}\right) \geq \kappa$. Hence $u_{n}^{+}$must be nonvanishing. Let us define $v_{n}=u_{n}\left(\cdot-s_{n}\right)$. Then

$$
\lim _{n \rightarrow \infty} \int_{B_{r}(0)}\left(v_{n}^{+}\right)^{2} d t \geq \frac{\delta}{2}
$$

Since $I_{\lambda}$ and $I_{\lambda}^{\prime}$ are both invariant under translation, we know that

$$
I_{\lambda}^{\prime}\left(v_{n}\right) \rightarrow 0, \quad I_{\lambda}\left(v_{n}\right) \rightarrow c_{\lambda}
$$

Since $v_{n}$ is still bounded, we may assume that $v_{n}^{+} \rightarrow u_{\lambda}^{+}$and $v_{n}^{-} \rightarrow u_{\lambda}^{-}$. From Lemma 2.1 we know that $v_{n}^{+} \rightarrow u_{\lambda}^{+}$in $L_{l o c}^{2}\left(\mathbb{R}, \mathbb{R}^{2 n}\right)$, which together with (2.7) implies $u_{\lambda}^{+} \neq 0$. Note that

$$
\left(I_{\lambda}^{\prime}\left(u_{\lambda}\right), \varphi\right)=\lim _{n \rightarrow \infty}\left(I_{\lambda}^{\prime}\left(v_{n}\right), \varphi\right)=0, \forall \varphi \in D(\mathbb{R}) .
$$

Applying $\left(W_{4}\right)$ and Fatou's lemma, we have

$$
\begin{aligned}
\sup _{\bar{Q}} I \geq c_{\lambda} & =\lim _{n \rightarrow \infty}\left(I_{\lambda}\left(v_{n}\right)-\frac{1}{2}\left(I_{\lambda}^{\prime}\left(v_{n}\right), v_{n}\right)\right) \\
& =\lim _{n \rightarrow \infty} \lambda \int_{\mathbb{R}}\left(\frac{1}{2}\left(\nabla W\left(t, v_{n}\right), v_{n}\right)-W\left(t, v_{n}\right)\right) d t \\
& \geq \lambda \int_{\mathbb{R}}\left(\frac{1}{2}\left(\nabla W\left(t, u_{\lambda}\right), u_{\lambda}\right)-W\left(t, u_{\lambda}\right)\right) d t=I_{\lambda}\left(u_{\lambda}\right) ;
\end{aligned}
$$

thus we get $I_{\lambda}\left(u_{\lambda}\right) \leq \sup _{\bar{Q}} I$.

To continue the discussion, we need the following two lemmas:

Lemma 2.6 ([3]). Let $u, v \in \mathbb{R}^{2 n}, s \in \mathbb{R}$ with $s \geq 1$ and $w:=s u+v \neq 0$, and let $t \in \mathbb{R}$. If $\left(W_{0}\right)$ and $\left(W_{4}\right)-\left(W_{6}\right)$ are satisfied, then

$$
\left(\nabla W(t, u), s\left(\frac{s}{2}-1\right) u+(s-1) v\right)+W(t, u)-W(t, w-u) \leq 0 .
$$

Lemma 2.7. Letting $\left\{u_{\lambda}\right\}$ be the critical point of $I_{\lambda}$ obtained in Lemma 2.5, we have

$$
I_{\lambda}\left(w-u_{\lambda}\right) \leq I_{\lambda}\left(u_{\lambda}\right) \text { for any } w \in \Sigma:=\left\{s u_{\lambda}+v: s \geq 1, v \in E^{-}\right\}, w \neq 0 .
$$

Proof. We rewrite $I_{\lambda}$ by

$$
I_{\lambda}\left(u_{\lambda}\right)=\frac{1}{2}\left(B u_{\lambda}^{+}, u_{\lambda}^{+}\right)+\frac{\lambda}{2}\left(B u_{\lambda}^{-}, u_{\lambda}^{-}\right)-\lambda \int_{\mathbb{R}} W\left(t, u_{\lambda}\right) d t .
$$

Since $I_{\lambda}^{\prime}\left(u_{\lambda}\right)=0$, we have

$$
\begin{aligned}
0= & \left(I_{\lambda}^{\prime}\left(u_{\lambda}\right), s\left(\frac{s}{2}-1\right) u_{\lambda}+(s-1) v\right) \\
= & s\left(\frac{s}{2}-1\right)\left(B u_{\lambda}^{+}, u_{\lambda}^{+}\right)+\lambda s\left(\frac{s}{2}-1\right)\left(B u_{\lambda}^{-}, u_{\lambda}^{-}\right)+\lambda(s-1)\left(B u_{\lambda}^{-}, v\right) \\
& -\lambda \int_{\mathbb{R}}\left(\nabla W\left(t, u_{\lambda}\right), s\left(\frac{s}{2}-1\right) u_{\lambda}+(s-1) v\right) d t,
\end{aligned}
$$


which together with the fact that $w=s u_{\lambda}+v,(2.10)$ and Lemma 2.6 implies that

$$
\begin{aligned}
& I_{\lambda}\left(w-u_{\lambda}\right)-I_{\lambda}\left(u_{\lambda}\right) \\
&=\frac{1}{2}\left\{\left(B(s-1) u_{\lambda}^{+},(s-1) u_{\lambda}^{+}\right)-\left(B u_{\lambda}^{+}, u_{\lambda}^{+}\right)\right\} \\
&+\frac{\lambda}{2}\left\{\left(B\left((s-1) u_{\lambda}^{-}+v\right),(s-1) u_{\lambda}^{-}+v\right)-\left(B u_{\lambda}^{-}, u_{\lambda}^{-}\right)\right\} \\
&+\lambda\left\{\int_{\mathbb{R}} W\left(t, u_{\lambda}\right) d t-\int_{\mathbb{R}} W\left(t, w-u_{\lambda}\right) d t\right\} \\
&=s\left(\frac{s}{2}-1\right)\left(B u_{\lambda}^{+}, u_{\lambda}^{+}\right)+\lambda s\left(\frac{s}{2}-1\right)\left(B u_{\lambda}^{-}, u_{\lambda}^{-}\right)+\frac{\lambda}{2}(B v, v)+\lambda(s-1)\left(B u_{\lambda}^{-}, v\right) \\
&+\lambda\left\{\int_{\mathbb{R}} W\left(t, u_{\lambda}\right) d t-\int_{\mathbb{R}} W\left(t, w-u_{\lambda}\right) d t\right\} \\
&=\lambda \int_{\mathbb{R}}\left[\left(\nabla W\left(t, u_{\lambda}\right), s\left(\frac{s}{2}-1\right) u_{\lambda}+(s-1) v\right)+W\left(t, u_{\lambda}\right)-W\left(t, w-u_{\lambda}\right)\right] d t \\
&+\frac{\lambda}{2}(B v, v) \\
& \leq 0 .
\end{aligned}
$$

So Lemma 2.7 holds.

Lemma 2.8. Under assumptions $\left(L_{0}\right)$ and $\left(W_{0}\right)-\left(W_{6}\right)$, there exist $\lambda_{n} \rightarrow 1$ and $a$ sequence $\left\{u_{\lambda_{n}}\right\}$ such that

$$
I_{\lambda_{n}}^{\prime}\left(u_{\lambda_{n}}\right)=0, \quad I_{\lambda_{n}}\left(u_{\lambda_{n}}\right) \leq \sup _{\bar{Q}} I
$$

Moreover $\left\{u_{\lambda_{n}}\right\}$ is bounded.

Proof. The existence of $\left\{u_{\lambda_{n}}\right\}$ such that

$$
I_{\lambda_{n}}^{\prime}\left(u_{\lambda_{n}}\right)=0, \quad I_{\lambda_{n}}\left(u_{\lambda_{n}}\right) \leq \sup _{\bar{Q}} I
$$

is the direct consequence of Lemma 2.5. To prove the boundedness of $\left\{u_{\lambda_{n}}\right\}$, arguing by contradiction, suppose that $\left\|u_{\lambda_{n}}\right\| \rightarrow \infty$. Since $I_{\lambda_{n}}\left(u_{\lambda_{n}}\right) \geq 0$, we know that $\left\|u_{\lambda_{n}}^{+}\right\| \geq\left\|u_{\lambda_{n}}^{-}\right\|$. Let $v_{\lambda_{n}}:=\frac{u_{\lambda_{n}}}{\left\|u_{\lambda_{n}}\right\|}$. Then $\left\|v_{\lambda_{n}}^{+}\right\|^{2} \geq \frac{1}{2}$ and $v_{\lambda_{n}} \rightarrow v$ a.e. in $\mathbb{R}$, after passing to a subsequence. Since $\left\{v_{\lambda_{n}}\right\}$ is bounded, we have that either $v_{\lambda_{n}}^{+}$is vanishing, i.e.,

$$
\lim _{n \rightarrow \infty} \sup _{s \in \mathbb{R}} \int_{B_{1}(s)}\left(v_{\lambda_{n}}^{+}\right)^{2} d t=0
$$

or it is nonvanishing; i.e., there exist $r, \delta>0$ and a sequence $s_{n} \in \mathbb{R}$ such that

$$
\lim _{n \rightarrow \infty} \int_{B_{r}\left(s_{n}\right)}\left(v_{\lambda_{n}}^{+}\right)^{2} d t \geq \delta .
$$

If $v_{\lambda_{n}}^{+}$is vanishing, by Lion's concentration compactness principle, we have that $v_{\lambda_{n}}^{+} \rightarrow 0$ in $L^{q}\left(\mathbb{R}, \mathbb{R}^{2 n}\right)$ for all $q \in(2, \infty)$. By Hölder's inequality and (2.4) we have that $\int_{\mathbb{R}} W\left(t, R v_{\lambda_{n}}^{+}\right) d t \rightarrow 0$ for any $R \in \mathbb{R}$. Note that Lemma 2.7 implies that 
$I_{\lambda_{n}}\left(u_{\lambda_{n}}\right) \geq I_{\lambda_{n}}\left(R v_{\lambda_{n}}^{+}\right)$for any $R \geq 0$. Therefore, we have

$$
\begin{aligned}
\sup _{\bar{Q}} I \geq I_{\lambda_{n}}\left(u_{\lambda_{n}}\right) \geq I_{\lambda_{n}}\left(R v_{\lambda_{n}}^{+}\right) & =\frac{R^{2}}{2}\left\|v_{\lambda_{n}}^{+}\right\|^{2}-\lambda_{n} \int_{\mathbb{R}} W\left(t, R v_{\lambda_{n}}^{+}\right) d t \\
& \geq \frac{R^{2}}{4}-2 \int_{\mathbb{R}} W\left(t, R v_{\lambda_{n}}^{+}\right) d t \rightarrow \frac{R^{2}}{4},
\end{aligned}
$$

so we arrive at a contradiction if $R$ is large enough. Hence $v_{\lambda_{n}}^{+}$must be nonvanishing and the invariance of $I_{\lambda_{n}}$ under translation implies that $s_{n}$ can be selected to be bounded. Therefore, by Lemma 2.1 we know that $v_{\lambda_{n}}^{+} \rightarrow v_{\lambda}^{+}$in $L_{\text {loc }}^{2}\left(\mathbb{R}, \mathbb{R}^{2 n}\right)$, which together with (2.11) implies that $v_{\lambda}^{+} \neq 0$. So $\left|u_{\lambda_{n}}\right| \rightarrow \infty$ as $n \rightarrow \infty$. It follows again from $\left(W_{3}\right)$ and Fatou's lemma that

$$
\int_{\mathbb{R}} \frac{W\left(t, u_{\lambda_{n}}\right)}{u_{\lambda_{n}}^{2}} v_{\lambda_{n}}^{2} d t \rightarrow \infty \text { as } n \rightarrow \infty
$$

Thus

$$
0 \leq \frac{I_{\lambda}\left(u_{\lambda_{n}}\right)}{\left\|u_{\lambda_{n}}\right\|^{2}}=\frac{1}{2}\left\|v_{\lambda_{n}}^{+}\right\|^{2}-\lambda\left(\frac{1}{2}\left\|v_{\lambda_{n}}^{-}\right\|^{2}+\int_{\mathbb{R}} \frac{W\left(t, u_{\lambda_{n}}\right)}{u_{\lambda_{n}}^{2}} v_{\lambda_{n}}^{2} d t\right) \rightarrow-\infty \text { as } n \rightarrow \infty,
$$

which is a contradiction. Thus we have the conclusion.

Lemma 2.9. If $\left\{u_{\lambda_{n}}\right\}$ is the sequence obtained in Lemma 2.8, then it is also a $(P S)$ sequence for I satisfying

$$
\lim _{n \rightarrow \infty} I^{\prime}\left(u_{\lambda_{n}}\right)=0, \quad \lim _{n \rightarrow \infty} I\left(u_{\lambda_{n}}\right) \leq \sup _{\bar{Q}} I .
$$

Proof. Since $u_{\lambda_{n}}$ is bounded, from

$$
\lim _{n \rightarrow \infty} I\left(u_{\lambda_{n}}\right)=\lim _{n \rightarrow \infty}\left(I_{\lambda_{n}}\left(u_{\lambda_{n}}\right)+\left(\lambda_{n}-1\right)\left(\frac{1}{2}\left\|u_{\lambda_{n}}^{-}\right\|^{2}+\int_{\mathbb{R}} W\left(t, u_{\lambda_{n}}\right) d t\right)\right)
$$

and noting that

$$
\begin{aligned}
& \lim _{n \rightarrow \infty}\left(I^{\prime}\left(u_{\lambda_{n}}\right), \varphi\right) \\
& =\lim _{n \rightarrow \infty}\left(\left(I_{\lambda_{n}}^{\prime}\left(u_{\lambda_{n}}\right), \varphi\right)+\left(\lambda_{n}-1\right)\left(\left(u_{\lambda_{n}}^{-}, \varphi^{-}\right)+\int_{\mathbb{R}}\left(\nabla W\left(t, u_{\lambda_{n}}\right), \varphi\right) d t\right)\right)
\end{aligned}
$$

uniformly in $\|\varphi\| \leq 1$, we obtain the conclusion.

\section{Proof of main Result}

We are now in a position to prove our Theorem 1.3.

Proof. Since $\left\{u_{\lambda_{n}}\right\}$ is bounded, we have that either $u_{\lambda_{n}}$ is vanishing, i.e.,

$$
\lim _{n \rightarrow \infty} \sup _{s \in \mathbb{R}} \int_{B_{1}(s)} u_{\lambda_{n}}^{2} d t=0,
$$

or it is nonvanishing; i.e., there exist $r, \delta>0$ and a sequence $s_{n} \in \mathbb{R}$ such that

$$
\lim _{n \rightarrow \infty} \int_{B_{r}\left(s_{n}\right)} u_{\lambda_{n}}^{2} d t \geq \delta
$$


If $u_{\lambda_{n}}$ is vanishing, by Lion's concentration compactness principle, we have that $u_{\lambda_{n}} \rightarrow 0$ in $L^{q}\left(\mathbb{R}, \mathbb{R}^{2 n}\right)$ for all $q \in(2, \infty)$. However, since $\left(I_{\lambda_{n}}^{\prime}\left(u_{\lambda_{n}}\right), u_{\lambda_{n}}^{+}\right)=0$, and by Hölder's inequality, the Sobolev embedding theorem and (2.3), we know that

$$
\begin{aligned}
\left\|u_{\lambda_{n}}^{+}\right\|^{2} & =\lambda \int_{\mathbb{R}}\left(\nabla W\left(t, u_{\lambda_{n}}\right), u_{\lambda_{n}}^{+}\right) d t \\
& \leq \varepsilon \int_{\mathbb{R}}\left|u_{\lambda_{n}}\right| \cdot\left|u_{\lambda_{n}}^{+}\right| d t+C_{\varepsilon} \int_{\mathbb{R}}\left|u_{\lambda_{n}}\right|^{p-1}\left|u_{\lambda_{n}}^{+}\right| d t \\
& \leq \varepsilon\left\|u_{\lambda_{n}}\right\| \cdot\left\|u_{\lambda_{n}}^{+}\right\|+C_{\varepsilon}^{\prime}\left\|u_{\lambda_{n}}\right\|_{p}^{p-1}\left\|u_{\lambda_{n}}^{+}\right\| \\
& \leq \varepsilon\left\|u_{\lambda_{n}}\right\| \cdot\left\|u_{\lambda_{n}}^{+}\right\|+C_{\varepsilon}^{\prime \prime}\left\|u_{\lambda_{n}}\right\|_{p}^{p-2}\left\|u_{\lambda_{n}}\right\| \cdot\left\|u_{\lambda_{n}}^{+}\right\| \\
& \leq \varepsilon\left\|u_{\lambda_{n}}\right\|^{2}+C_{\varepsilon}^{\prime \prime}\left\|u_{\lambda_{n}}\right\|_{p}^{p-2}\left\|u_{\lambda_{n}}\right\|^{2} .
\end{aligned}
$$

Similarly, we have

$$
\left\|u_{\lambda_{n}}^{-}\right\|^{2} \leq \varepsilon\left\|u_{\lambda_{n}}\right\|^{2}+C_{\varepsilon}^{\prime \prime}\left\|u_{\lambda_{n}}\right\|_{p}^{p-2}\left\|u_{\lambda_{n}}\right\|^{2} .
$$

From (3.3) and (3.4), we get

$$
\left\|u_{\lambda_{n}}\right\|^{2} \leq 2 \varepsilon\left\|u_{\lambda_{n}}\right\|^{2}+2 C_{\varepsilon}^{\prime \prime}\left\|u_{\lambda_{n}}\right\|_{p}^{p-2}\left\|u_{\lambda_{n}}\right\|^{2},
$$

which means that $\left\|u_{\lambda_{n}}\right\|_{p} \geq c$ for some constant $c$; hence (3.1) does not hold. Let us define $v_{\lambda_{n}}=u_{\lambda_{n}}\left(\cdot-s_{n}\right)$. Then

$$
\lim _{n \rightarrow \infty} \int_{B_{r}(0)}\left(v_{\lambda_{n}}\right)^{2} d t \geq \frac{\delta}{2}
$$

Since $I_{\lambda}$ and $I_{\lambda}^{\prime}$ are both invariant under translation, we know that

$$
I_{\lambda}^{\prime}\left(v_{\lambda_{n}}\right) \rightarrow 0
$$

Since $v_{\lambda_{n}}$ is still bounded, we may assume that $v_{\lambda_{n}} \rightarrow u$. From Lemma 2.1 we know that $v_{\lambda_{n}} \rightarrow u$ in $L_{l o c}^{2}\left(\mathbb{R}, \mathbb{R}^{2 n}\right)$, which together with (3.5) implies $u \neq 0$ with $I^{\prime}(u)=0$.

Let $K:=\left\{u \in E: I^{\prime}(u)=0\right\}$ be the critical set of $I$ and let

$$
C:=\inf \{I(z): z \in K \backslash\{0\}\} .
$$

For any critical point $u$ of $I$, assumption $\left(W_{4}\right)$ implies that

$$
I(u)=I(u)-\frac{1}{2}\left(I^{\prime}(u), u\right)=\int_{\mathbb{R}}\left[\frac{1}{2}(\nabla W(t, u), u)-W(t, u)\right] d t>0 \text { if } u \neq 0 .
$$

Therefore $C \geq 0$. We prove that $C>0$ and that there is $u \in K$ such that $I(u)=C$. Let $u_{j} \in K \backslash\{0\}$ be such that $I\left(u_{j}\right) \rightarrow C$. Then, the proof in Lemma 2.8 shows that $\left(u_{j}\right)$ is bounded, and by the concentration compactness principle discussion above we know that $u_{j} \rightarrow u \in K \backslash\{0\}$. Thus

$$
\begin{aligned}
C=\lim _{j \rightarrow \infty} I\left(u_{j}\right) & =\lim _{j \rightarrow \infty} \int_{\mathbb{R}}\left(\frac{1}{2}\left(\nabla W\left(t, u_{j}\right), u_{j}\right)-W\left(t, u_{j}\right)\right) d t \\
& \geq \int_{\mathbb{R}}\left(\frac{1}{2}(\nabla W(t, u), u)-W(t, u)\right) d t=I(u) \geq C,
\end{aligned}
$$

the first inequality being due to $\left(W_{4}\right)$ and Fatou's lemma. So $I(u)=C$ and $C>0$ because $u \neq 0$. 


\section{REFERENCES}

1. G. Arioli, A. Szulkin, Homoclinic solutions of Hamiltonian systems with symmetry, J. Differential Equations 158 (1999), 291-313. MR.1721901 (2000k:37084)

2. V. Coti-Zelati, I. Ekeland, E. Séré, A variational approach to homoclinic orbits in Hamiltonian systems, Math. Ann. 288 (1990), 133-160. MR1070929 (91g:58065)

3. G. Chen, S. Ma, Periodic solutions for Hamiltonian systems without Ambrosetti-Rabinowitz condition and spectrum 0, J. Math. Anal. Appl. 379 (2011), 842-851.

4. Y.H. Ding, M. Girardi, Infinitely many homoclinic orbits of a Hamiltonian system with symmetry, Nonlinear Anal. 38 (1999), 391-415. MR1705761 (2000f:34087)

5. Y.H. Ding, M. Willem, Homoclinic orbits of a Hamiltonian system, Z. Angew. Math. Phys. 50 (1999), 759-778. MR1721793 (2000k:37086)

6. Y.H. Ding, Multiple homoclinics in a Hamiltonian system with asymptotically or super linear terms, Commun. Contemp. Math. 8 (2006), 453-480. MR2258874 (2007h:37092)

7. Y.H. Ding, Variational Methods for Strongly Indefinite Problems, Interdiscip. Math. Sci., vol. 7, World Scientific, 2007. MR2389415 (2009e:58014)

8. H. Hofer, K. Wysocki, First order elliptic systems and the existence of homoclinic orbits in Hamiltonian systems, Math. Ann. 228 (1990), 483-503. MR.1079873 (91m:58064)

9. L. Jeanjean, On the existence of bounded Palais-Smale sequences and application to a Landesman-Lazer type problem set on $\boldsymbol{R}^{N}$, Proc. Roy. Soc. Edinburgh Sect. A 129 (1999), 787-809. MR1718530 (2001c:35034)

10. P.L. Lions, The concentration-compactness principle in the calculus of variations: The locally compact cases, Part I, AIP Anal. Non Linéaire 1 (1984), 109-145; Part II, AIP Anal. Non Linéaire 1 (1984), 223-283. MR778970 (87e:49035a); MR778974 (87e:49035b)

11. E. Séré, Existence of infinitely many homoclinic orbits in Hamiltonian systems, Math. Z. 209 (1992), 27-42. MR1143210 (92k:58201)

12. E. Séré, Looking for the Bernoulli shift, Ann. Inst. H. Poincaré, Anal. Non Linéaire 10 (1993), 561-590. MR1249107 (95b:58031)

13. M. Struwe, Variational Methods: Applications to Nonlinear Partial Differential Equations and Hamiltonian Systems, Springer-Verlag, Berlin, 2000. MR.1736116 (2000i:49001)

14. A. Szulkin, W. Zou, Homoclinic orbits for asymptotically linear Hamiltonian systems, J. Funct. Anal. 187 (2001), 25-41. MR.1867339 (2002h:37127)

15. M. Schechter, W. Zou, Weak linking theorems and Schrödinger equations with critical Sobolev exponent, ESAIM Control Optim. Calc. Var. 9 (2003), 601-619. MR1998717 (2004h:35078)

16. A. Szulkin, T. Weth, Ground state solutions for some indefinite variational problems, J. Funct. Anal. 257 (2009), 3802-3822. MR2557725 (2010j:35193)

17. K. Tanaka, Homoclinic orbits in a first order super-quadratic Hamiltonian system: convergence of subharmonic orbits, J. Differential Equations 94 (1991), 315-339. MR1137618 (93e:58072)

18. M. Willem, Minimax Theorems, Birkhäuser, Boston, 1996. MR.1400007 (97h:58037)

19. M. Willem, W. Zou, On a Schrödinger equation with periodic potential and spectrum point zero, Indiana Univ. Math. J. 52 (2003), 109-132. MR.1970023 (2004a:35082)

20. X. Xu, Homoclinic orbits for first order Hamiltonian systems possessing super-quadratic potentials, Nonlinear Anal. 51 (2002), 197-214. MR1918340(2003m:37089)

21. M. Yang, W. Chen, and Y. Ding, Solutions for discrete periodic Schrödinger equations with spectrum 0, Acta Appl. Math. 110 (3) (2010), 1475-1488. MR2639182 (2011d:39002)

22. M. Yang, W. Chen, and Y. Ding, Solutions for periodic Schrödinger equation with spectrum zero and general superlinear nonlinearities, J. Math. Anal. Appl. 364 (2) (2010), 404-413. MR2576192 (2011b:35167)

23. M. Yang, Ground state solutions for a periodic Schrödinger equation with superlinear nonlinearities, Nonlinear Anal. 72 (5) (2010), 2620-2627. MR2577823(2011a:35186)

24. S. Zhang, Symmetrically homoclinic orbits for symmetric Hamiltonian systems, J. Math. Anal. Appl. 247 (2000), 645-652. MR:1769099 (2001f:37101)

School of Mathematical Sciences and LPMC, Nankai University, Tianjin 300071, PeoPLE'S RePublic of ChinA

E-mail address: guanweic@163.com

E-mail address: shiwangm@163.net 